\title{
BMJ Open Underlying mechanisms of complex interventions addressing the care of older adults with multimorbidity: a realist review
}

\author{
Monika Kastner, ${ }^{1,2,3}$ Leigh Hayden, ${ }^{1}$ Geoff Wong, ${ }^{4}$ Yonda Lai, ${ }^{3}$ Julie Makarski, ${ }^{1}$ \\ Victoria Treister, ${ }^{3}$ Joyce Chan, ${ }^{1}$ Julianne H Lee, ${ }^{3}$ Noah M Ivers, ${ }^{5,6}$ \\ Jayna Holroyd-Leduc, ${ }^{7}$ Sharon E Straus ${ }^{3,8}$
}

To cite: Kastner M, Hayden L, Wong G, et al. Underlying mechanisms of complex interventions addressing the care of older adults with multimorbidity: a realist review. BMJ Open 2019;9:e025009. doi:10.1136/ bmjopen-2018-025009

- Prepublication history and additional material for this paper are available online. To view these files, please visit the journal online (http://dx.doi. org/10.1136/bmjopen-2018025009).

Received 10 July 2018 Revised 25 January 2019 Accepted 26 February 2019

Check for updates

(C) Author(s) (or their employer(s)) 2019. Re-use permitted under CC BY-NC. No commercial re-use. See rights and permissions. Published by BMJ.

For numbered affiliations see end of article.

Correspondence to Dr Monika Kastner; monika.kastner@utoronto.ca

\section{ABSTRACT}

Objectives To understand how and why effective multichronic disease management interventions influence health outcomes in older adults 65 years of age or older. Design A realist review.

Data sources Electronic databases including Medline and Embase (inception to December 2017); and the grey literature.

Eligibility criteria for selecting studies We considered any studies (ie, experimental quasi-experimental, observational, qualitative and mixed-methods studies) as long as they provided data to explain our programme theories and effectiveness review (published elsewhere) findings. The population of interest was older adults (age $\geq 65$ years) with two or more chronic conditions.

Analysis We used the Realist And MEta-narrative Evidence Syntheses: Evolving Standards (RAMESES) quality and publication criteria for our synthesis aimed at refining our programme theories such that they contained multiple context-mechanism-outcome configurations describing the ways different mechanisms fire to generate outcomes. We created a 3-step synthesis process grounded in metaethnography to separate units of data from articles, and to derive explanatory statements across them.

Results 106 articles contributed to the analysis. We refined our programme theories to explain multimorbidity management in older adults: (1) care coordination interventions with the best potential for impact are teambased strategies, disease management programmes and case management, (2) optimised disease prioritisation involves ensuring that clinician work with patients to identify what symptoms are problematic and why, and to explore options that are acceptable to both clinicians and patients and (3) optimised patient self-management is dependent on patients' capacity for selfcare and to what extent, and establishing what patients need to enable selfcare.

Conclusions To optimise care, both clinical management and patient self-management need to be considered from multiple perspectives (patient, provider and system). To mitigate the complexities of multimorbidity management, patients focus on reducing symptoms and preserving quality of life while providers focus on the condition that most threaten morbidity and mortality.

PROSPERO registration number CRD42014014489.
Strengths and limitations of this study

- To our knowledge, this is the first realist review to explain why multimorbidity interventions work, for whom, and under what circumstances to improve outcomes for older adults with multimorbidity findings can be used to inform practice and policy decisions in the management of older adults with multiple chronic conditions

- Our search strategy was in part informed by a systematic review investigating the effectiveness of multimorbidity interventions for older adults that we conducted alongside this realist review.

- We created a 3-step synthesis process drawn from meta-ethnography to separate units of data from articles, and to derive explanatory statements across them.

- Many of our included studies did not have complete data to enable optimised investigation of context-mechanism-outcome (CMO) configurations.

- Incomplete reporting also impacted our ability to fully test our theories and therefore, we could not completely elucidate the interrelationships within and between all of our CMO configurations.

\section{BACKGROUND}

The global population is ageing, with 2 billion people expected to reach 60 years of age and older by $2050 .{ }^{12}$ It is now more common for older adults to have multiple chronic diseases than to have single diseases or no chronic medical conditions at all. ${ }^{3}$ The burden of chronic disease is also on the rise globally ${ }^{14}$ with more than half of older adults (age $\geq 65$ years) living with high-burden chronic conditions (ie, highly prevalent and associated with premature death and increased healthcare utilisation). ${ }^{3}{ }^{5}$ Older adults also have greater healthcare needs, are at higher risk for adverse health outcomes, and experience more frequent hospitalisations, ${ }^{6}$ yet only $55 \%$ receive appropriate care. ${ }^{78}$ In response, 
different chronic disease management $(\mathrm{CDM})$ interventions have been created. For example, a programme designed to encourage older adults with Chronic Obstructive Pulmonary Disease (COPD) and depression to adhere to antidepressants and pulmonary rehabilitation. ${ }^{9}$ Although promising, CDM interventions have shown varying effectiveness ${ }^{10}{ }^{11}$ in part, because they are not usually developed for older adults or created for sustained use; and very few are designed to deliberately address multimorbidity. ${ }^{812}$

Given our rapidly ageing population, there is an urgent need to understand how and why multimorbidity interventions influence health outcomes to optimise patient care. To address these gaps, we conducted a systematic review to identify effective CDM interventions that integrate the care of $\geq 2$ high-burden chronic diseases affecting older adults (published elsewhere) ${ }^{13}$ However, a systematic review is not always enough to inform practice and policy decisions as knowing 'what' works seldom reveals which desired outcomes may occur under different contexts. Our objective was to conduct a realist review alongside to explore the underlying mechanisms and contexts by which these CDM interventions work or do not work, for whom, under what circumstances and why. ${ }^{14}$ Realist review is particularly relevant for making sense of complex interventions (such as those focusing on CDM) that have context-sensitive outcomes. It can add important contextual and mechanistic detail to existing knowledge on this topic. ${ }^{15}$ Such detail is likely to contribute to the limited existing clinical practice guidelines on multi-morbidity management such as those developed by National Institute for Health and Care Excellence (NICE), ${ }^{16}$ by explaining the contexts in which intended and unintended outcomes are likely to occur. Additional resources about realist reviews can be found the RAMESES Project website. ${ }^{17}$ Our overall objective of this review is to: understand how and why effective CDM interventions influence health outcomes in older adults 65 years of age or older.

\section{METHODS}

\section{Study design}

Our protocol was published ${ }^{18}$ and registered with PROSPERO registration number. We applied the RAMESES quality ${ }^{19}$ and reporting criteria. ${ }^{20}$ The systematic review methods and findings are reported elsewhere. ${ }^{13}$

\section{Programme theory development}

To identify our initial programme theories (ie, what multimorbidity interventions are composed of, how and why they are expected to work and what outcomes they might generate), we used an iterative, consensus-based process. We considered two major sources to identify any published or unpublished literature ${ }^{21}$ : (1) Medline and Google Scholar describing models, frameworks, theories of multimorbidity, CDM and complex interventions and (2) content and methods experts on our team (geriatricians, family physicians, and health services and realist review experts). Duplicate screening of 97 reports by two reviewers identified 18 documents that contained data that helped us to understand CDM interventions. Through team discussion and a Delphi survey among our team, we identified that our initial programme theory would have to incorporate the following concepts: (1) CDM interventions are complex interventions that do provide different outcomes in different settings; (2) health prioritisation is an important aspect of multimorbidity and (3) interventions that consider patient values and circumstances, the evidence and the clinician's expertise were more likely to produce desired outcomes. We then used the data from our included studies to gradually refine our understanding of these concepts and how (if at all) they fit into our more refined programme theory developed from this review.

\section{Search strategy}

Since we performed our realist review alongside our systematic review of multimorbidity interventions, ${ }^{13}$ the search strategy was done simultaneously for both reviews. As such, we identified potentially relevant articles for our realist review (ie, to provide data to test our programme theories) through our systematic review search strategy (inception to December 2017) ${ }^{13}$ and performed additional iterative, targeted searches as needed for the realist review. ${ }^{19}$ An experienced information specialist performed these additional searches in Medline and Embase (online supplementary appendix 1).

\section{Selection and appraisal of documents}

To increase the efficiency of our searching and screening process, reviewer pairs independently screened titles and abstracts simultaneously for both the systematic review and realist review. We considered any study design for inclusion (ie, experimental quasi-experimental, observational, qualitative and mixed-methods studies). During full-text screening, we considered all articles that were identified for the systematic review as well through additional targeted searches to explain our programme theories and effectiveness review findings. Two reviewers independently assessed each article for relevance (does the source contain any data that could be interpreted as having our relevant context, mechanism or outcome for programme theory development?) and rigour (How trustworthy are the data? Does the article provide enough detail on how conclusions were reached irrespective of study design?).

\section{Data extraction}

We created and pilot tested a standardised data extraction form. Data items were driven by our purpose to refine our programme theories through context-mechanism-outcome (CMO) configurations (ie, if we were able to infer an explanation for the cause $[\mathrm{M}]$ for a particular outcome $[\mathrm{O}]$ under the influence of one or more particular contexts [C]). For example, computer-based counselling systems (intervention) targeting older adults and providers in primary care $(\mathrm{C})$ are not acceptable $(\mathrm{O})$ if 
they do not show any relative advantage over the current system $\left(\mathrm{M}_{1}\right)$ and if inconsistent with providers' current practice workflow $\left(\mathrm{M}_{2}\right)$. After extracting excerpts in duplicate, reviewer pairs independently assigned an associated concept code and iteratively developed a codebook of concepts (online supplementary appendix 1) that was used to code subsequent excerpts; any discrepancies were discussed and resolved as a team.

\section{Analysis and synthesis processes}

We used the RAMESES quality ${ }^{19}$ and publication ${ }^{20}$ criteria to guide the synthesis. Our goal was to refine our programme theories such that they contained multiple CMO configurations describing the ways different mechanisms fire to generate outcomes. We created a 3-step synthesis process grounded in meta-ethnography ${ }^{22}$ to separate units of data from articles, and to derive explanatory statements across them. Step 1: Reviewer pairs independently extracted relevant excerpts from articles. Step 2: One reviewer sorted excerpts by concept for each study and developed consolidated statements (groups of CMO configurations) for each. A second reviewer audited the first reviewer's statements by checking for agreement and consistency with their own interpretations. Step 3: As a team, we examined and compared consolidated statements across studies to derive explanatory statements. These were then used to refine our programme theories aimed at explaining the outcome patterns we found within the effectiveness review. When the consolidated statements seemed to disagree, we unpacked the concepts and further examined them, consulting our literature and content experts as necessary for additional data and insights.

\section{Deviations from our protocol in conducting our realist review}

We followed the methods as outlined in our protocol ${ }^{18}$ with a few exceptions. First, we switched to an auditing process during Step 2 of the analysis to make our process more efficient. This involved an auditor checking the work of a primary reviewer. Second, since our process to finalise the list of initial programme theories identified an area that was not covered by our systematic review search (ie, health prioritisation), we added a secondary search strategy to capture this literature as described above.

\section{Patient and public involvement}

Patients were not involved in the conduct of the review but older adults with multiple chronic conditions are involved in developing key messages for this research. These patients are also part of our broader integrated knowledge translation team to co-design an electronic self-management tool that integrates the care of multiple chronic conditions (KeepWell); this tool is being informed by this review.

\section{RESULTS}

\section{Study characteristics}

Figure 1 is our Preferred Reporting Items for Systematic Reviews and Meta-Analyses diagram, which shows the flow of article selection. Of 2435 potentially, relevant citations that were screened for relevance, 124 articles were reviewed in full-text, and 106 articles contributed to the analysis. ${ }^{3} 915$ 23-125 Studies were published between 2002 and 2016 mostly in the USA $(n=32)$, the UK $(n=19)$, Canada $(n=14)$, Germany $(n=11)$ and Australia $(n=10)$. Most of the articles $(75 \%)$ were about multimorbidity $(\mathrm{n}=50)$ or disease prioritisation $(\mathrm{n}=29)$, and 27 studies $(25 \%)$ addressed specific chronic disease combinations.

\section{Programme theories}

Using data from our included studies, we iteratively developed and refined our initial two programme theories and a third programme theory that emerged from our data. To make our findings more succinct, in the following paragraphs, we have provided narratives that summarise the most important aspects of our programme theories. This approach obscures the detailed CMO configurations that underpin these narratives and may make our manuscript less useful for those interested in realist review methodology. To address this issue, we have provided indications of the CMO configurations that our narratives are based on. For those interested in seeing the links between our data and CMO configurations, please see online supplementary appendices 3-6 that explains the outcomes that may be achieved by the different intervention strategies used in care coordination under different contexts.

\section{Programme theory 1: Care coordination interventions for} multimorbidity management

Almost one-half of the interventions described in our realist review were 'care coordination' interventions (ie, changes in how healthcare workers interact with each other or patients to ensure timely and efficient delivery of healthcare). ${ }^{126}$ Online supplementary appendix 3 shows their detailed $\mathrm{CMO}$ configurations that underpin this programme theory. Overall, we found that care coordination interventions in primary care are effective for older adults with multimorbidity because they represent a structured approach to holistic care. They address multiple conditions through interdisciplinary teams or multidisciplinary disease management, providing specific processes for communication and establishing formal roles for providers and patients. We identified three types of care coordination approaches that healthcare providers may wish to use that have potential for impact: (1) Team-based or collaborative approaches involve highly trained clinicians ${ }^{53}$ providing holistic and coordinated care ${ }^{88}$ including spending time with patients to discuss all their concerns, and to prevent care overlap and gaps. ${ }^{80}$ Patients are given education, counselling and other support services to address their disease(s), medications, and lifestyle. ${ }^{44}$ Team-based approaches can provide access to specialist ${ }^{53}$ and a wider range of services, and provide evidence-based care solutions for multiple conditions in parallel (not in tandem). ${ }^{38}$ Optimised care outcomes are most likely to occur through interdisciplinary communication and collaboration, ${ }^{38} 81$ when teams comprise highly trained and skilled members ${ }^{53}$ who understand and accept 


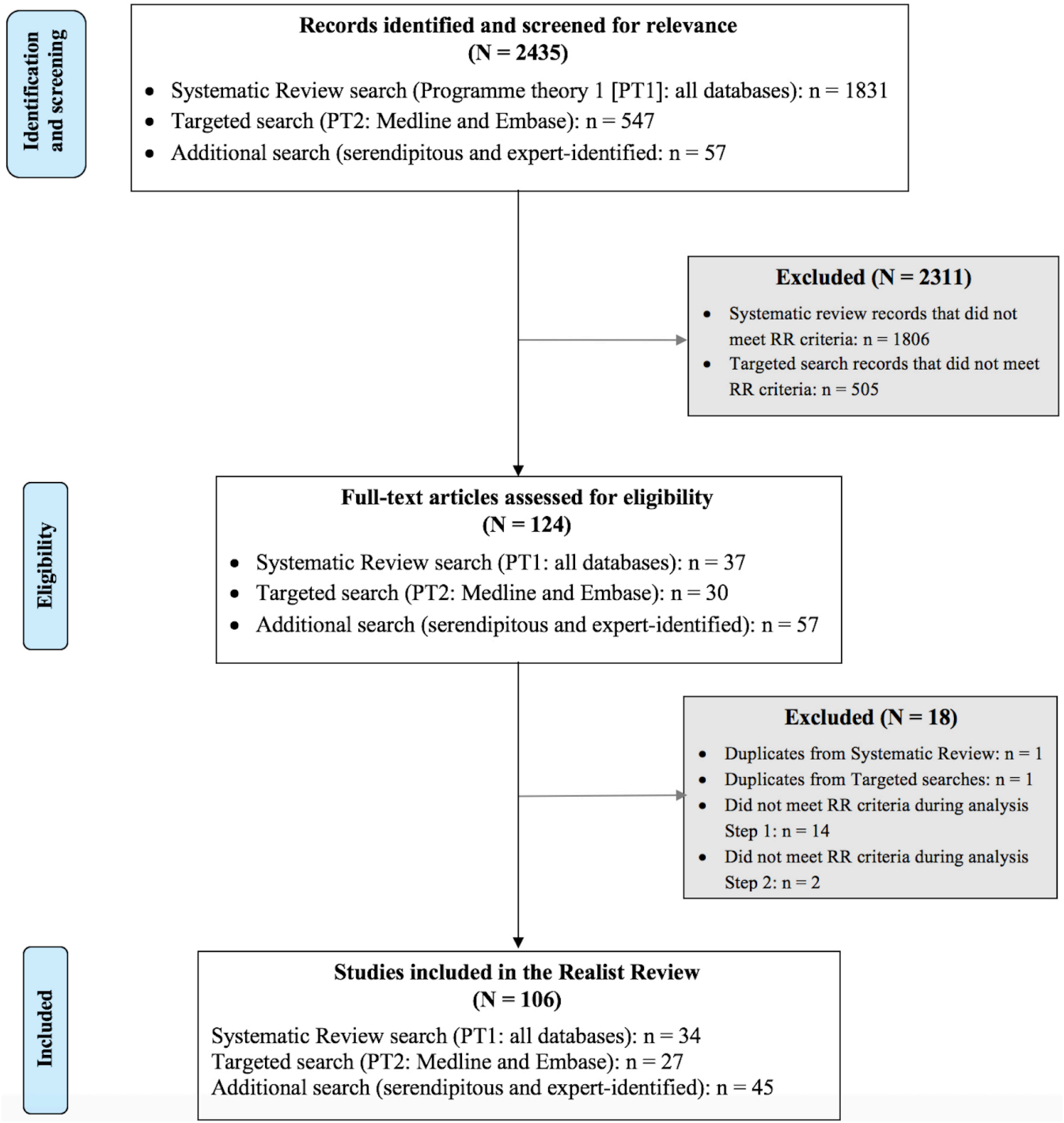

Figure 1 Flow of article selection for the Realist Review.

each other's roles, ${ }^{53}$ provide opportunities ${ }^{3888}$ and time ${ }^{53}$ to share information, ${ }^{81}$ and collaborate on patient care. ${ }^{3845} 5388$ Other contexts in which mechanisms are likely to be triggered include teams that have dedicated members who provide additional support to patients ${ }^{38} 53$ or providers, ${ }^{81}$ receive training, ${ }^{38} 5381$ and have a robust and well-functioning communication system. ${ }^{38} 45$ (2) Disease management programmes follow a 'script' for how to provide effective patient care via care protocols or plans, which define the division of tasks, support the follow-up and coordination of action, ${ }^{103} 110$ and help to sustain a philosophy of common care. ${ }^{45}$ Systematised care is achieved through checklists, follow-up timetables ${ }^{45} 103110$ and treatment targets, ${ }^{45}$ which can lead to a shared philosophy of care ${ }^{45103}$ and optimised decision making. ${ }^{45}$ (3) Case management: Case managers are trained healthcare professionals who are the main contact (and conduit of information) between a patient and involved providers, ${ }^{53}$ and most appropriate for multimorbidity management when there may be multiple and diverse providers involved in a patient's care. When case managers are the primary contact, ${ }^{80} 103$ care is perceived by patients as continuous, ${ }^{78} 79$ coordinated ${ }^{79}$ and more individualised, ${ }^{980}$ and fosters the development of the skills and confidence patients need to self-manage their health. ${ }^{78}$

\section{Programme theory 2: Disease prioritisation in multimorbidity} management

The detailed CMO configurations of disease prioritisation that underpin this programme theory are described in online supplementary appendix 4. Multimorbidity management is perceived as confusing for patients and overwhelming for providers due to the heterogeneous nature of multimorbidity, ${ }^{102}$ disease and treatment interactions and possible conflicts, ${ }^{57} 92$ and the difficulty of attributing symptoms to conditions. ${ }^{57}$ Multimorbidity can create a cognitive and emotional overload in patients and providers, ${ }^{64}$ so a common strategy they use is to focus on one condition at a time. Patients and providers focus their attention by prioritising one condition over another for a specified period of time, or until particular outcomes are achieved. ${ }^{6491}$ However, patients and providers approach prioritisation differently. Patients 
make prioritisation judgements based on the symptoms they experience and need the most attention. They identify the most undesired symptoms and focus on their associated condition(s) ${ }^{3256636668125}$ or those that threaten their social activities, ${ }^{256376}$ limit their independence ${ }^{2591}$ and have potentially severe long-term consequences if not addressed. ${ }^{6391}$ Providers prioritise conditions based on their judgements about the prognosis or severity of the condition and place greater emphasis on conditions with more serious outcomes 2557666876125 ; they focus on conditions that threaten a patient's morbidity and mortality, ${ }^{25} 576668125$ those they think they are better equipped to address (eg, physical over emotional ${ }^{32} 124$ ), and whether the patient is likely to benefit from treatment. ${ }^{57} 114124125$ What's common among patients and providers, is that they both consider conditions that they feel capable of addressing, ${ }^{6491} 124125$ and both consider the cascading effects of multimorbidity and the interrelatedness of these conditions during the prioritisation process. ${ }^{65}{ }^{91}$ For patients, the cascading effects of multimorbidity are particularly challenging. Patients may find it difficult to determine which chronic disease is causing a particular symptom because conditions may share similar symptoms ${ }^{72}$ or the treatment of one condition may aggravate the other ${ }^{61} 629091$ or cause other antagonistic effects. ${ }^{649091}$ Self-management is therefore a challenge for patients because the diagnosis of (and receipt of information) about a new condition compounds the complexity and uncertainty of what to do. ${ }^{87}$ Figure 2 shows our conceptualisation of optimised disease prioritisation from the perspective of providers and patients. For this simplified overall programme theory, we have analysed and interpreted our findings in such a way as to provide a programme theory that presents out findings in a more familiar format using the concepts of 'barriers' and 'facilitators'. The programme theory sets out the factors that need to be taken into account if providers and patients wish to optimise disease prioritisation. In particular we provide an overview of factors that healthcare providers may need to address to help patients to: (1) identify what symptoms are bothering them; (2) why they bother them and (3) exploring options that are acceptable to them for addressing their symptoms.

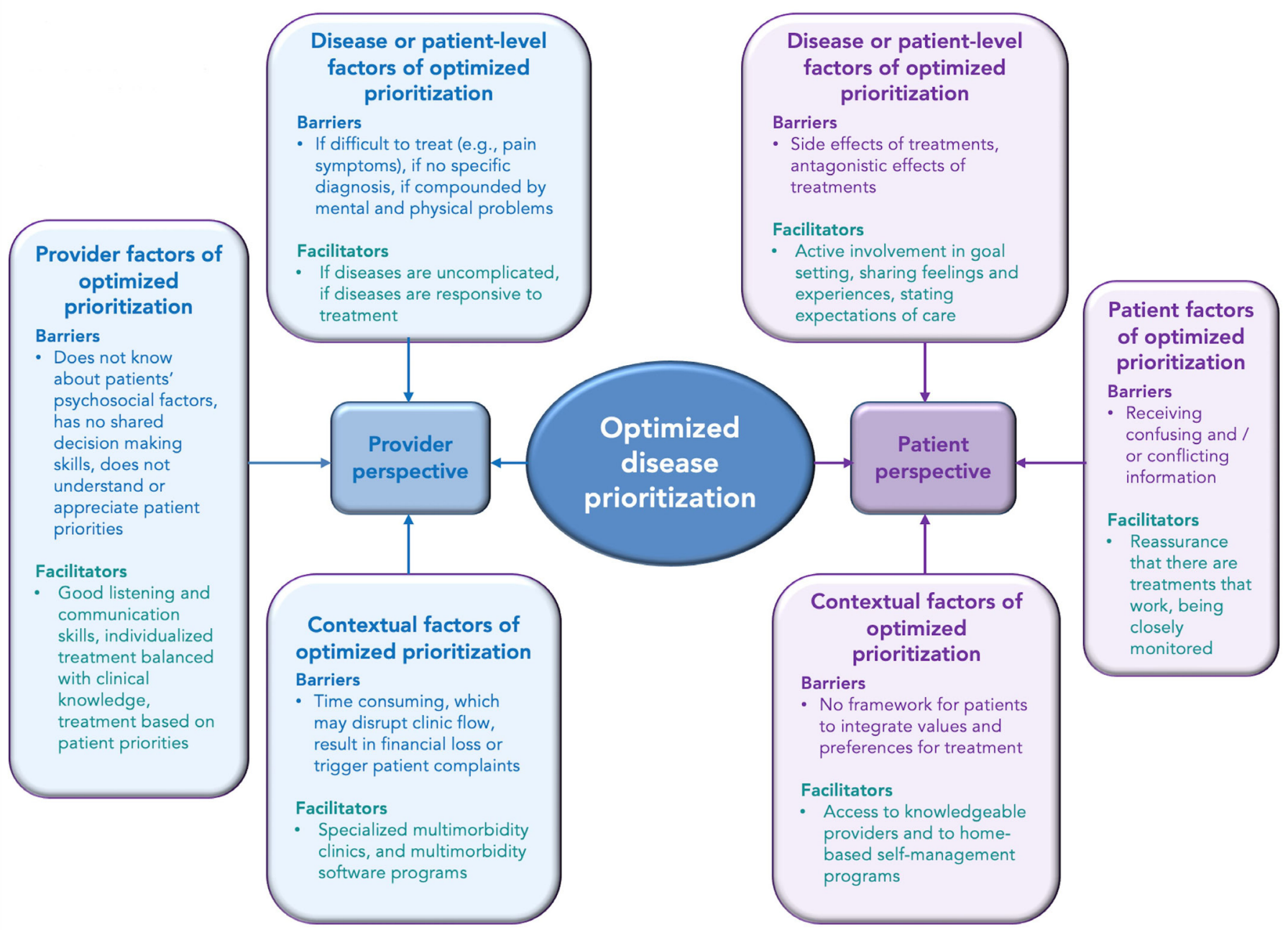

Figure 2 Framework of optimised disease prioritization in multimorbidity management. A simplified overarching programme theory of identifying factors (conceptualised as barriers and facilitators) that need to be considered by patients and providers when trying to optimise disease prioritization. 
Programme theory 3: Patient self-management in multimorbidity The detailed CMO configurations of multimorbidity self-management that underpin this programme theory are in online supplementary appendix 5. Multimorbidity is perceived by patients as a burden because of the volume of information and recommendations provided ${ }^{5174}$ which are often inconsistent or conflicting, and the cognitive and emotional overload required to assimilate this information or to make lifestyle changes. ${ }^{87}$ Subsequently, this can lead to confusion and non-adherence to recommendations $^{254391-93}$ and may also trigger cognitive and emotional overload. Specific explanations to these outcomes include (1) self-management regimens are designed to fit their condition rather than their health priorities, lifestyle and available resources ${ }^{89} 94$; (2) prescribed medications are unwieldy (too many, taken often, and difficult to keep track of $)^{1551}$ or mismanaged $^{71}$; (3) difficulties with following the required diet and exercise routine ${ }^{36} 51$ 91; and to see multiple providers ${ }^{71}$; (4) not knowing how to respond to adverse drug effects ${ }^{1571}$ and (5) experiencing communication barriers due to linguistic and cultural diversity. ${ }^{71}$ Self-management is especially challenging for older adults with cognitive impairment ${ }^{89}$ or anxiety ${ }^{90}$ in addition to other chronic conditions, as these contexts can interact to increase people's perceived illness burden. ${ }^{63} \mathrm{In}$ particular, if depression is the additional condition, older adults may choose not to do anything at all because they either consider it a normal part of ageing or reluctant to seek treatment due to the stigma associated with mental health problems. ${ }^{30}$ Depression, as a context, can therefore also trigger additional mechanisms that reduce a patient's ability to self-manage chronic conditions ${ }^{30-32} 59648791$ : reduced motivation, energy, self-efficacy and feelings of hopelessness, ${ }^{31}$ and stress. ${ }^{87}$ A number of feedback loops are activated because illness burden can interfere with a person's ability to engage in health promotion (eg, exercise). This can lead to negative consequences (eg, weight gain, ${ }^{87}$ reduced quality of life, functional decline), and in turn impair mood, social networks, and self-management behaviours. ${ }^{62}$ Multimorbidity self-management is also influenced by the lack of available resources ${ }^{64}(\mathrm{eg}$, adequate finances, ${ }^{6291}$ social supports ${ }^{236288} 8991$ or transportation $^{91}$ ) or low health literacy ${ }^{29}$ or skills to manage adverse effects. ${ }^{4390}$ Older adults are interested in self-management tools that provide health condition information $^{51}$; share, coordinate and synthesise information with and between providers; and connect them with other patients. ${ }^{51}$ Physicians can support this by tailoring information to the stage of the patient's condition, ${ }^{26}$ having interactions with patients, ${ }^{93}$ providing information ${ }^{93}$ and fostering a collaborative approach to care. ${ }^{115}$

\section{DISCUSSION}

In this realist review we developed and refined our programme theories to explain why coordination of care interventions (found to have the most potential for impact in our systematic review) work to improve outcomes for older adults with multimorbidity. Care coordination interventions may be effective in primary care because they represent a structured approach to comprehensive care, and address multiple conditions through interdisciplinary teams or multidisciplinary disease management by providing specific processes for communication, and establishing formal roles for providers and patients. Teambased approaches provide the right care at the right time, disease management offers a systematised approach to care, and case management offers a dedicated case manager as the conduit of care.

In addition to refining our programme theories, we generated explanations associated with these theories. Online supplementary appendix 6 shows the CMO configurations to explain of multimorbidity management overall. Figure 3 shows our conceptualisation of multimorbidity management, which suggests that optimised care requires both clinical management and patient self-management, with the caveat that each needs to consider identified challenges from the perspective of those affected by them (patient, provider, system). From the patient perspective, clinical management can be confusing due to conflicting messages, which is compounded in the presence of depression, impaired cognition, or poor health literacy. The mental health needs of patients can further complicate clinical management by impeding selfcare, creating communication barriers with providers (eg, patient complaints may not be clear), and patients receiving less intensive treatment. Self-management is difficult for patients because of the high burden of required lifestyle changes and adherence to multiple and often conflicting treatment regimens. Multimorbidity can also have cascading effects due to the nature of how chronic diseases are interrelated and the influence of a patient's mental and emotional health on self-management. From the provider perspective, clinical management of multimorbidity may be perceived as overwhelming because of the heterogeneous nature of multimorbidity, and conflicting or lack of evidence to guide clinical decision making. Lack of skills and confidence, not having decision support systems and protocols that are too rigid can also lead to inadequate preparation to manage multimorbidity. From a system perspective, even if primary care is the optimal setting for multimorbidity management, it may not always have the infrastructure to support optimal strategies such as care coordination and can also lead to fragmentation of care.

\section{Recommendations}

Findings from programme theory 1 suggests that healthcare providers may wish to use care coordination interventions that are: (1) Team-based or collaborative approaches that involve highly trained clinicians providing holistic and coordinated care through effective interdisciplinary communication and collaboration, and the provision of education and counselling to patients to address their disease(s), medications and lifestyle; (2) Disease management programmes via care protocols or plans, checklists, 


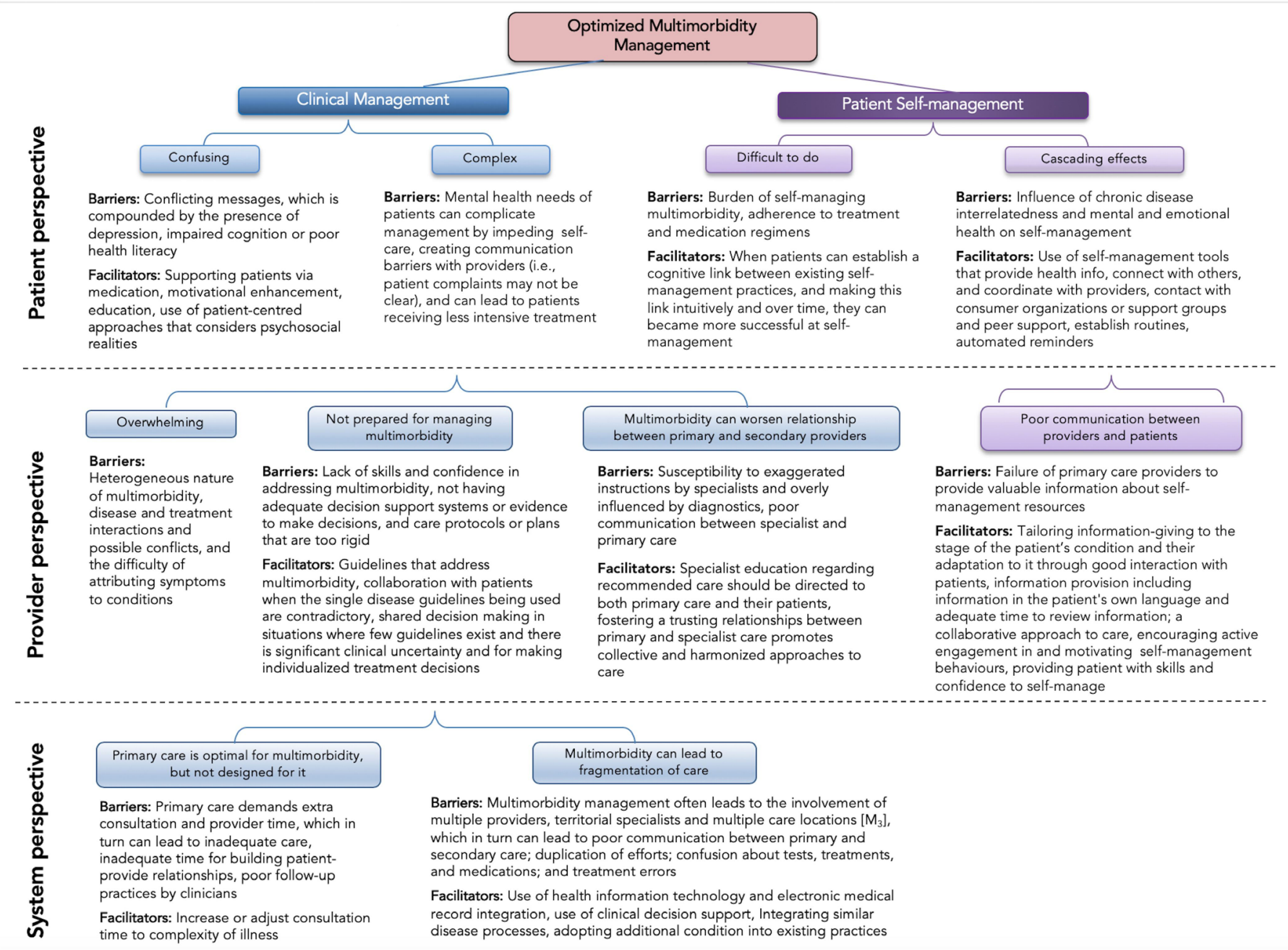

Figure 3 Framework of optimised multimorbidity management. A simplified overarching programme theory identifying factors (conceptualised as barriers and facilitators) that need to be considered when trying to optimise multimorbidity management from the patient, provider and system perspective.

follow-up timetables and treatment targets and (3) Case management strategies for situations when there may be multiple and diverse providers involved in a patient's care. For programme theory 2, the specific types of disease prioritisation approach that healthcare providers may wish to consider is to work with patients to identify what symptoms are bothering them and why, and exploring options that are acceptable to both clinicians and patients for addressing their symptoms. For programme theory 3, the specific types of self-management approach that healthcare providers may wish to consider include not assuming that all patients are capable of selfcare, identifying who is capable of selfcare and to what extent, and establishing with the patient what they need (eg, information, support) to enable selfcare.

\section{Strengths and limitations}

To our knowledge, this is the first realist review investigating older adult multimorbidity aimed at explaining why effective multi-CDM interventions (identified through a systematic review ${ }^{13}$ ) work/do not work for whom, under what circumstances and why. This can better inform practice and policy decisions about multimorbidity management than a systematic review alone. A Cochrane review investigated interventions in multimorbid patients of any age $\mathrm{e}^{15}$ and found mixed results, but concluded that interventions that were integrated with care and targeted specific risk factors or functional difficulties may be more effective. ${ }^{15}$ A rapid realist review investigating the underlying mechanisms of care planning strategies found that the mechanisms driving positive outcomes for people with long-term conditions are those that motivate them and promote an understanding of their role in self-management and how their lifestyle affects their conditions. ${ }^{127}$ Our findings build on these studies by providing explanations for why multimorbidity interventions may be effective for older adults. Additionally, we focused exclusively on older adults because they represent a relatively unstudied population, and given their projected population growth, they urgently need our attention to optimise their care. NICE guidelines on clinical assessment and management of multimorbidity ${ }^{16}$ (one of few existing multimorbidity guidelines) support 
many of our findings. They emphasise the need to find synergies in care regimes and simplifying care where possible. They also describe a preferred approach to care, which involves establishing patient goals, values and priorities, where patients are encouraged to describe their preferred decision making approach and what aspects of their life they prioritise. ${ }^{16} \mathrm{~A}$ recent qualitative systematic review also highlights the need for providers to simplify the burden of care for multimorbid patients. ${ }^{128}$ Our findings highlight the importance of focusing multimorbidity management by prioritising one or more specific condition(s) and ensuring that prioritisation is undertaken in collaboration with patients.

Our study has some limitations. First, it is possible that other teams may have identified different programme theories or interpretations. However, we used a rigorous and systematic process, and we let our data guide our interpretations. Second, many of our included studies did not have complete data to enable optimised CMO investigations. This may in part be due to an over-emphasis on effectiveness research in the literature, and an under-representation of qualitative inquiry, particularly about elucidating 'mechanisms'. For example, the literature rarely addressed the social determinants of health (a potentially significant trigger for multimorbidity outcomes) even though many older adults experience social isolation ${ }^{129}$ and financial ${ }^{130}$ challenges. Incomplete reporting also impacted our ability to fully test our theories. As such, while we developed and refined a number of explanations for our data, we could not completely elucidate the interrelationships within and between all of our CMO configurations. Finally, it is important to note that since this analysis was interpretive and inductive, it is possible that another team of researchers would have arrived at a different set of programme theories that incorporate the mechanisms and contexts of multi-CDM interventions for older adults. Thus, these findings should only be used as potential mid-range theories to explore and interrogate.

\section{CONCLUSIONS AND FUTURE DIRECTIONS}

Our realist review contributes to the current, limited knowledge of the underlying mechanisms of complex multi-CDM interventions for older adults with multimorbidity. We found that care coordination interventions are effective because they represent a structured approach to holistic care. To mitigate the complexities of multimorbidity management, patients focus on reducing their undesired symptoms and preserving their quality of life, while providers focus on the condition that most threaten a patient's morbidity and mortality. To optimise care, multimorbidity management requires both clinical management and patient self-management, and be considered from multiple perspectives (patient, provider and system).

\section{Author affiliations}

${ }^{1}$ Knowledge Translation and Implementation, Research and Innovation, North York General Hospital, Toronto, Ontario, Canada
${ }^{2}$ Institute of Health Policy, Management and Evaluation, University of Toronto, Toronto, Ontario, Canada

${ }^{3}$ Li Ka Shing Knowledge Institute, St. Michael's Hospital, Toronto, Ontario, Canada ${ }^{4}$ Nuffield Department of Primary Care Health Sciences, University of Oxford, Oxford, UK

${ }^{5}$ Family and Community Medicine, University of Toronto, Toronto, Ontario, Canada ${ }^{6}$ Family Medicine, Women's College Hospital, Toronto, Ontario, Canada

${ }^{7}$ Medicine, Cumming School of Medicine, University of Calgary, Calgary, Alberta, Canada

${ }^{8}$ Medicine, University of Toronto, Toronto, Ontario, Canada

Acknowledgements In addition to our core research team, we would like to thank Becky Skidmore and Alissa Epworth for helping to develop and execute the search strategies for this review. We would also like to thank our Patient and Family Advisory Council members at North York General Hospital in Toronto, Ontario, who are helping to support the dissemination of findings from this review and are using findings to codesign a multimorbidity self-management tool for older adults (KeepWell).

Contributors MK: Manuscript development and final approval, methods design, data acquisition, data extraction, data analysis, research question development. LH: Manuscript development and final approval, data extraction, data analysis. GW: Manuscript development and final approval, methods design and data interpretation. YL: Manuscript development and final approval, data extraction, data analysis, methods. JM: Manuscript development and final approval, data extraction, data analysis, methods. VT: Manuscript development and final approval, data extraction, data analysis, methods design. JC: Manuscript development and final approval, data extraction, data analysis. JL: Manuscript development and final review, data extraction, data analysis. NMI: Manuscript development and final approval, methods design, data acquisition. JH-L: Manuscript development and final approval, methods design, data acquisition. SES: Manuscript development and final approval, methods design, data acquisition.

Funding This research was supported by an Ontario, Canada Ministry of Health and Long-term Care (MOHLTC) Health Systems Research Fund (HSRF) Capacity Award. The funder was not involved in conducting the realist review. Monika Kastner is funded by a Canadian Institutes of Health Research (CIHR) New Investigator Award. Geoff Wong is partly funded by The Evidence Synthesis Working Group of the United Kingdom's National Institute for Health Research School for Primary Care Research (NIHR SPCR) [Project Number 390]. Noah Ivers is funded by a CIHR New Investigator Award and a Clinician Scientist Award from the Department of Family and Community Medicine, University of Toronto. Jayna Holroyd-Leduc is funded by a University of Calgary BSF Chair in Geriatric Medicine. Sharon Straus is funded by a Tier 1 Canada Research Chair in Knowledge Translation.

Competing interests None declared.

Patient consent for publication Not required.

Provenance and peer review Not commissioned; externally peer reviewed.

Data sharing statement We included most of the data generated or analysed for this study in this published article and associated appendices. Any additional datasets are available from the corresponding author upon request.

Open access This is an open access article distributed in accordance with the Creative Commons Attribution Non Commercial (CC BY-NC 4.0) license, which permits others to distribute, remix, adapt, build upon this work non-commercially, and license their derivative works on different terms, provided the original work is properly cited, appropriate credit is given, any changes made indicated, and the use is non-commercial. See: http://creativecommons.org/licenses/by-nc/4.0/.

\section{REFERENCES}

1. Chatterji S, Byles J, Cutler D, et al. Health, functioning, and disability in older adults - present status and future implications. The Lancet 2015;385:563-75.

2. Statistics_Canada. Canada Yearbook. Seniors. 2012 http://www. statcan.gc.ca/pub/11-402-x/2012000/chap/seniors-aines/seniorsaines-eng.htm (Accessed 8th May 2017).

3. Marengoni A, Angleman S, Melis R, et al. Aging with multimorbidity: a systematic review of the literature. Ageing Res Rev 2011;10:430-9.

4. Yach D, Hawkes C, Gould CL, et al. The global burden of chronic diseases: overcoming impediments to prevention and control. JAMA 2004;291:2616-22. 
5. WHO. NCDs | Noncommunicable diseases and their risk factors. WHO Web site: World Health Organization. Accessed. http://www. who.int/ncds/en/. Published 2018. Updated 2018-03-20 14:55:41

6. Boyd C, Fortin M. Future of multimorbidity research: How should understanding of multimorbidity inform health system design? Public Health Reviews 2011;33:451-74.

7. Moore EG, Rosenberg MW, Fitzgibbon SH. Activity limitation and chronic conditions in Canada's elderly, 1986-2011. Disabil Rehabil 1999;21:196-210

8. Ward BW, Schiller JS. Prevalence of multiple chronic conditions among US adults: estimates from the National Health Interview Survey, 2010. Prev Chronic Dis 2013;10:E65.

9. Alexopoulos GS, Kiosses DN, Sirey JA, et al. Untangling therapeutic ingredients of a personalized intervention for patients with depression and severe COPD. Am J Geriatr Psychiatry 2014;22:1316-24.

10. Weingarten SR, et al. Interventions used in disease management programmes for patients with chronic illness---which ones work? Meta-analysis of published reports. BMJ 2002;325:925.

11. Wenger NS, Solomon DH, Roth CP, et al. The quality of medical care provided to vulnerable community-dwelling older patients. Ann Intern Med 2003;139:740-7.

12. Coleman K, Austin BT, Brach C, et al. Evidence on the chronic care model in the new millennium. Health Aff 2009;28:75-85.

13. Kastner M, Cardoso R, Lai Y, et al. Effectiveness of interventions for managing multiple high-burden chronic diseases in older adults: a systematic review and meta-analysis. CMAJ 2018;190:E101 2:E1004-

14. Greenhalgh T, Peacock R. Effectiveness and efficiency of search methods in systematic reviews of complex evidence: audit of primary sources. BMJ 2005;331:1064-5.

15. Smith SM, Wallace E, O'Dowd T, et al. Interventions for improving outcomes in patients with multimorbidity in primary care and community settings. Cochrane Database Syst Rev 2016;3:CD006560.

16. Centre NG. (Great Britain), National Institute for Health and Care Excellence (Great Britain). Multimorbidity: assessment, prioritisation, and management of care for people with commonly occurring multimorbidity: clinical assessment and management. NICE guideline: methods, evidence and recommendations NG56. London: National Institute for Health and Care Excellence, 2016.

17. Project R. The RAMESES Projects. $2013 \mathrm{http}: / / \mathrm{www}$. ramesesproject.org/ (Accessed 29 November, 2018.)

18. Kastner M, Perrier L, Hamid J, et al. Effectiveness of knowledge translation tools addressing multiple high-burden chronic diseases affecting older adults: protocol for a systematic review alongside a realist review. BMJ Open 2015;5:e007640.

19. Wong G, Greenhalgh T, Westhrop G, et al. Development of methodological guidance, publication standards and training materials for realist and meta-narrative reviews: the RAMESES (Realist And Meta-narrative Evidence Syntheses 12013 Evolving Standards) project. Southampton, UK: NIHR Journals Library, 2014.

20. Wong G, Greenhalgh T, Westhorp G, et al. RAMESES publication standards: realist syntheses. BMC Med 2013;11:21.

21. Pawson R, Greenhalgh T, Harvey G, et al. Realist review--a new method of systematic review designed for complex policy interventions. J Health Serv Res Policy 2005;10:21-34.

22. Noblit G, Hare R. Meta-ethnography: Synthesizing qualitative studies. Newbury Park, CA: Sage, 1988.

23. Webster F, Christian J, Mansfield E, et al. Capturing the experiences of patients across multiple complex interventions: a metaqualitative approach. BMJ Open 2015;5:e007664.

24. Sun $X$, Guyatt GH. Interventions to enhance self management support. BMJ 2013;346:f3949.

25. Junius-Walker $U$, Voigt I, Wrede J, et al. Health and treatment priorities in patients with multimorbidity: report on a workshop from the european general practice network meeting 'research on multimorbidity in general practice'. Eur J Gen Pract 2010;16:51-4

26. Infante FA, Proudfoot JG, Powell Davies G, et al. How people with chronic illnesses view their care in general practice: a qualitative study. Med J Aust 2004;181:70-3.

27. Naik $A D$, White CD, Robertson SM, et al. Behavioral health coaching for rural-living older adults with diabetes and depression: an open pilot of the HOPE Study. BMC Geriatr 2012;12:37

28. Lamers $\mathrm{F}$, Jonkers $\mathrm{CC}$, Bosma $\mathrm{H}$, et al. A minimal psychological intervention in chronically ill elderly patients with depression: a randomized trial. Psychother Psychosom 2010;79:217-26.

29. Kenning C, Protheroe J, Gray N, et al. The potential for using a Universal Medication Schedule (UMS) to improve adherence in patients taking multiple medications in the UK: a qualitative evaluation. BMC Health Serv Res 2015;15:94.

30. Unützer J, Hantke M, Powers D, et al. Care management for depression and osteoarthritis pain in older primary care patients: a pilot study. Int J Geriatr Psychiatry 2008;23:1166-71.

31. Cj W, Chang AM, Courtney M, et al. Peer supporters for cardiac patients with diabetes: a randomized controlled trial. Int Nurs Rev 2012;59:345-52.

32. Zulman DM, Kerr EA, Hofer TP, et al. Patient-provider concordance in the prioritization of health conditions among hypertensive diabetes patients. J Gen Intern Med 2010;25:408-14.

33. Kennedy A, Bower P, Reeves D, et al. Implementation of self management support for long term conditions in routine primary care settings: cluster randomised controlled trial. $B M J$ 2013;346:f2882.

34. McSweeney K, Jeffreys A, Griffith J, et al. Specialist mental health consultation for depression in Australian aged care residents with dementia: a cluster randomized trial. Int J Geriatr Psychiatry 2012;27:1163-71

35. Williams JW, Katon W, Lin EH, et al. The effectiveness of depression care management on diabetes-related outcomes in older patients. Ann Intern Med 2004;140:1015-24.

36. Eijkelberg IM, Mur-Veeman IM, Spreeuwenberg C, et al. Patient focus groups about nurse-led shared care for the chronically ill. Patient Educ Couns 2002;47:329-36.

37. Fraccaro P, Arguello Casteleiro M, Ainsworth J, et al. Adoption of clinical decision support in multimorbidity: a systematic review. JMIR Med Inform 2015;3:e4.

38. Knowles SE, Chew-Graham C, Adeyemi I, et al. Managing depression in people with multimorbidity: a qualitative evaluation of an integrated collaborative care model. BMC Fam Pract 2015;16:32.

39. Ricci-Cabello I, Violán C, Foguet-Boreu Q, et al. Impact of multimorbidity on quality of healthcare and its implications for health policy, research and clinical practice. A scoping review. Eur J Gen Pract 2015;21:192-202.

40. Smith SM, O'Kelly S, O'Dowd T. GPs' and pharmacists' experiences of managing multimorbidity: a 'Pandora's box'. British Journal of General Practice 2010;60:e285-94

41. Koné Pefoyo AJ, Bronskill SE, Gruneir A, et al. The increasing burden and complexity of multimorbidity. BMC Public Health 2015;15:415.

42. Brodaty H, Draper BM, Millar J, et al. Randomized controlled trial of different models of care for nursing home residents with dementia complicated by depression or psychosis. J Clin Psychiatry 2003;64:63-72.

43. Hammill AC, Wilson MG. Rapid synthesis: comparing multicomponent chronic-disease programs to disease-specific programs. Hamilton, Ontario: McMaster University, 2015.

44. Müller-Staub M, Zigan N, Händler-Schuster D, et al. [Being cared for and caring: living with multiple chronic diseases (Leila)-a qualitative study about APN contributions to integrated care]. Pflege 2015;28:79-91.

45. Lamothe L, Sylvain C, Sit V. [Multimorbidity and primary care: emergence of new forms of network organization]. Sante Publique 2015;27:S129-35.

46. Schnipper JL, Linder JA, Palchuk MB, et al. Effects of documentation-based decision support on chronic disease management. Am J Manag Care 2010;16:SP72-81.

47. Rahimpour M, Lovell NH, Celler BG, et al. Patients' perceptions of a home telecare system. Int J Med Inform 2008;77:486-98.

48. Bowles KH, Holland DE, Horowitz DA. A comparison of in-person home care, home care with telephone contact and home care with telemonitoring for disease management. $J$ Telemed Telecare 2009;15:344-50.

49. Whitten P, Mickus M. Home telecare for COPD/CHF patients: outcomes and perceptions. J Telemed Telecare 2007:13:69-73

50. Noel HC, Vogel DC, Erdos JJ, et al. Home telehealth reduces healthcare costs. Telemed J E Health 2004;10:170-83.

51. Zulman DM, Jenchura EC, Cohen DM, et al. How Can eHealth technology address challenges related to multimorbidity? perspectives from patients with multiple chronic conditions. J Gen Intern Med 2015;30:1063-70.

52. Becker A, Herzberg D, Marsden N, et al. A new computer-based counselling system for the promotion of physical activity in patients with chronic diseases--results from a pilot study. Patient Educ Couns 2011;83:195-202

53. Wozniak L, Soprovich A, Rees S, et al. Contextualizing the effectiveness of a collaborative care model for primary care patients with diabetes and depression (Teamcare): a qualitative assessment using RE-AIM. Can J Diabetes 2015;39:S83-91. 
54. Osborn R, Moulds D, Schneider EC, et al. Primary care physicians in ten countries report challenges caring for patients with complex health needs. Health Aff 2015;34:2104-12.

55. Williams A, Manias E, Walker R, et al. A multifactorial intervention to improve blood pressure control in co-existing diabetes and kidney disease: a feasibility randomized controlled trial. J Adv Nurs 2012;68:2515-25.

56. Muth C, van den Akker M, Blom JW, et al. The Ariadne principles: how to handle multimorbidity in primary care consultations. BMC Med 2014;12:223.

57. Luijks HD, Loeffen MJ, Lagro-Janssen AL, et al. GPs' considerations in multimorbidity management: a qualitative study. Br J Gen Pract 2012;62:e503-10.

58. Sinnott C, Mc Hugh S, Browne J, et al. GPs' perspectives on the management of patients with multimorbidity: systematic review and synthesis of qualitative research. BMJ Open 2013;3:e003610.

59. Bayliss EA, Edwards AE, Steiner JF, et al. Processes of care desired by elderly patients with multimorbidities. Fam Pract 2008;25:287-93.

60. Søndergaard E, Willadsen TG, Guassora AD, et al. Problems and challenges in relation to the treatment of patients with multimorbidity: General practitioners' views and attitudes. Scand J Prim Health Care 2015;33:121-6.

61. Smith SM, O'Dowd T. Chronic diseases: what happens when they come in multiples? Br J Gen Pract 2007;57:268-70.

62. Koch G, Wakefield BJ, Wakefield DS. Barriers and facilitators to managing multiple chronic conditions: a systematic literature review. West J Nurs Res 2015;37:498-516.

63. Cheraghi-Sohi S, Morden A, Bower P, et al. Exploring patient priorities among long-term conditions in multimorbidity: A qualitative secondary analysis. SAGE Open Med 2013;1:205031211350395.

64. Cheraghi-Sohi S, Bower P, Kennedy A, et al. Patient priorities in osteoarthritis and comorbid conditions: a secondary analysis of qualitative data. Arthritis Care Res 2013;65:920-7.

65. Bower P, Macdonald W, Harkness E, et al. Multimorbidity, service organization and clinical decision making in primary care: a qualitative study. Fam Pract 2011;28:579-87.

66. Löffler C, Altiner A, Streich W, et al. [Approaches of general practitioners and patients to multimorbidity. Qualitative study]. Z Gerontol Geriatr 2015;48:452-6.

67. Boult C, Karm L, Groves C. Improving chronic care: the "guided care" model. Perm J 2008;12:50-4.

68. Hansen $\mathrm{H}$, Pohontsch $\mathrm{N}$, van den Bussche $\mathrm{H}$, et al. Reasons for disagreement regarding illnesses between older patients with multimorbidity and their GPs - a qualitative study. BMC Fam Pract 2015;16:68.

69. Onder G, Palmer K, Navickas R, et al. Time to face the challenge of multimorbidity. A European perspective from the joint action on chronic diseases and promoting healthy ageing across the life cycle (JA-CHRODIS). Eur J Intern Med 2015;26:157-9.

70. van den Bussche $\mathrm{H}$, Koller D, Kolonko $\mathrm{T}$, et al. Which chronic diseases and disease combinations are specific to multimorbidity in the elderly? Results of a claims data based cross-sectional study in Germany. BMC Public Health 2011;11:101.

71. Williams A, Manias E, Liew D, et al. Working with CALD groups: testing the feasibility of an intervention to improve medication self management in people with kidney disease, diabetes,and cardiovascular disease. Renal Society of Australasia Journal 2012;8:62-9.

72. Zulman DM, Asch SM, Martins SB, et al. Quality of care for patients with multiple chronic conditions: the role of comorbidity interrelatedness. J Gen Intern Med 2014;29:529-37.

73. Sinnott C, Hugh SM, Boyce MB, et al. What to give the patient who has everything? A qualitative study of prescribing for multimorbidity in primary care. Br J Gen Pract 2015;65:e184-91.

74. Vogeli C, Shields AE, Lee TA, et al. Multiple chronic conditions: prevalence, health consequences, and implications for quality, care management, and costs. J Gen Intern Med 2007;22:391-5.

75. Wallace E, Salisbury C, Guthrie B, et al. Managing patients with multimorbidity in primary care. BMJ 2015;350:h176.

76. Junius-Walker U, Stolberg D, Steinke P, et al. Health and treatment priorities of older patients and their general practitioners: a crosssectional study. Qual Prim Care 2011;19:67-76.

77. Boyd CM, Boult C, Shadmi E, et al. Guided care for multimorbid older adults. Gerontologist 2007;47:697-704.

78. Hjelm M, Holmgren AC, Willman A, et al. Family members of older persons with multi-morbidity and their experiences of case managers in Sweden: an interpretive phenomenological approach. Int J Integr Care 2015;15:e011.
79. Hjelm M, Holst G, Willman A, et al. The work of case managers as experienced by older persons (75+) with multi-morbidity - a focused ethnography. BMC Geriatr 2015;15:168.

80. Spoorenberg SL, Wynia K, Fokkens AS, et al. Experiences of community-living older adults receiving integrated care based on the chronic care model: a qualitative study. PLoS One 2015;10:e0137803.

81. Lee L, Heckman G, McKelvie R, et al. Physicians' perceptions of capacity building for managing chronic disease in seniors using integrated interprofessional care models. Can Fam Physician 2015;61:e148-57.

82. Moffat K, Mercer SW. Challenges of managing people with multimorbidity in today's healthcare systems. BMC Fam Pract 2015;16:129.

83. Sinnige J, Braspenning J, Schellevis F, et al. The prevalence of disease clusters in older adults with multiple chronic diseases--a systematic literature review. PLoS One 2013;8:e79641.

84. Smith SM, Soubhi H, Fortin M, et al. Managing patients with multimorbidity: systematic review of interventions in primary care and community settings. BMJ 2012;345:e5205.

85. Coventry PA, Small N, Panagioti M, et al. Living with complexity; marshalling resources: a systematic review and qualitative metasynthesis of lived experience of mental and physical multimorbidity. BMC Fam Pract 2015;16:171.

86. Tinetti ME, Bogardus ST, Agostini JV. Potential pitfalls of diseasespecific guidelines for patients with multiple conditions. $N$ Engl $J$ Med 2004;351:2870-4

87. Lindsay S. Prioritizing illness: lessons in self-managing multiple chronic diseases. Canadian Journal of Sociology 2009;34:983-1002.

88. Tracy CS, Bell SH, Nickell LA, et al. The IMPACT clinic: innovative model of interprofessional primary care for elderly patients with complex health care needs. Can Fam Physician 2013;59:e148-55.

89. Fried TR, Tinetti ME, lannone L. Primary care clinicians' experiences with treatment decision making for older persons with multiple conditions. Arch Intern Med 2011;171:75-80.

90. Liddy C, Blazkho V, Mill K. Challenges of self-management when living with multiple chronic conditions: systematic review of the qualitative literature. Can Fam Physician 2014;60:1123-33.

91. Bratzke LC, Muehrer RJ, Kehl KA, et al. Self-management priority setting and decision-making in adults with multimorbidity: a narrative review of literature. Int J Nurs Stud 2015;52:744-55.

92. Harris MF, Dennis S, Pillay M. Multimorbidity: negotiating priorities and making progress. Aust Fam Physician 2013;42:850-4.

93. Morris RL, Sanders C, Kennedy AP, et al. Shifting priorities in multimorbidity: a longitudinal qualitative study of patient's prioritization of multiple conditions. Chronic IIIn 2011;7:147-61.

94. Dufour SP, Graham S, Friesen J, et al. Physiotherapists supporting self-management through health coaching: a mixed methods program evaluation. Physiother Theory Pract 2015;31:29-38.

95. Stellefson M, Chaney B, Barry AE, et al. Web 2.0 chronic disease self-management for older adults: a systematic review. J Med Internet Res 2013;15:e35.

96. Junius-Walker $U$, Wrede J, Schleef $\mathrm{T}$, et al. What is important, what needs treating? How GPs perceive older patients' multiple health problems: a mixed method research study. BMC Res Notes 2012;5:443.

97. Koroukian SM, Warner DF, Owusu C, et al. Multimorbidity redefined: prospective health outcomes and the cumulative effect of cooccurring conditions. Prev Chronic Dis 2015;12:E55.

98. Luijks H, Lucassen $\mathrm{P}$, van Weel $\mathrm{C}$, et al. How GPs value guidelines applied to patients with multimorbidity: a qualitative study. BMJ Open 2015;5:e007905.

99. Wrede J, Voigt I, Bleidorn J, et al. Complex health care decisions with older patients in general practice: patient-centeredness and prioritization in consultations following a geriatric assessment. Patient Educ Couns 2013;90:54-60.

100. Schäfer I, Kaduszkiewicz H, Wagner HO, et al. Reducing complexity: a visualisation of multimorbidity by combining disease clusters and triads. BMC Public Health 2014;14:1285.

101. Katon W, Unützer J, Fan MY, et al. Cost-effectiveness and net benefit of enhanced treatment of depression for older adults with diabetes and depression. Diabetes Care 2006;29:265-70.

102. Sinnige J, Korevaar JC, Westert GP, et al. Multimorbidity patterns in a primary care population aged 55 years and over. Fam Pract 2015;32:505-13.

103. Morgan MA, Coates MJ, Dunbar JA, et al. The TrueBlue mode of collaborative care using practice nurses as case managers for depression alongside diabetes or heart disease: a randomised trial. BMJ Open 2013;3:e002171. 
104. Lin EH, Katon W, Von Korff M, et al. Effect of improving depression care on pain and functional outcomes among older adults with arthritis: a randomized controlled trial. JAMA 2003;290:2428-9.

105. Bayliss EA. Simplifying care for complex patients. Ann Fam Med 2012;10:3-5

106. Foguet-Boreu Q, Violán C, Rodriguez-Blanco T, et al. Multimorbidity patterns in elderly primary health care patients in a south mediterranean European region: a cluster analysis. PLoS One 2015;10:e0141155

107. White KM, Terry DJ, Troup C, et al. An extended theory of planned behavior intervention for older adults with type 2 diabetes and cardiovascular disease. J Aging Phys Act 2012;20:281-99.

108. Bond CS, Worswick L. Self management and telehealth: lessons learnt from the evaluation of a dorset telehealth program. Patient 2015;8:311-6.

109. Bleich SN, Sherrod C, Chiang A, et al. Systematic review of programs treating high-need and high-cost people with multiple chronic diseases or disabilities in the United States, 2008-2014. Prev Chronic Dis 2015;12:E197.

110. Lemmens KM, Nieboer AP, Huijsman R. A systematic review of integrated use of disease-management interventions in asthma and COPD. Respir Med 2009;103:670-91.

111. Foret Giddens J, Tanner E, Frey K, et al. Expanding the gerontological nursing role in Guided Care. Geriatr Nurs 2009;30:358-64.

112. Palmer $\mathrm{C}$, Bycroft J, Healey $\mathrm{K}$, et al. Can formal collaborative methodologies improve quality in primary health care in New Zealand? Insights from the EQUIPPED Auckland Collaborative. J Prim Health Care 2012;4:328-36.

113. Martín-Lesende I, Orruño E, Bilbao A, et al. Impact of telemonitoring home care patients with heart failure or chronic lung disease from primary care on healthcare resource use (the TELBIL study randomised controlled trial). BMC Health Serv Res 2013;13:118.

114. Franek J. Self-management support interventions for persons with chronic disease: an evidence-based analysis. Ont Health Technol Assess Ser 2013;13:1-60.

115. Maly RC, Leake B, Frank JC, et al. Implementation of consultative geriatric recommendations: the role of patient-primary care physician concordance. J Am Geriatr Soc 2002;50:1372-80.

116. Jaglal SB, Guilcher SJ, Hawker G, et al. Impact of a chronic disease self-management program on health care utilization in rural communities: a retrospective cohort study using linked administrative data. BMC Health Serv Res 2014;14:198.
117. Kamerow D. How can we treat multiple chronic conditions? BMJ 2012;344:e1487.

118. Laiteerapong $\mathrm{N}$, Huang ES, Chin $\mathrm{MH}$. Prioritization of care in adults with diabetes and comorbidity. Ann N Y Acad Sci 2011;1243:69-87.

119. Arvidsson $\mathrm{E}$, André $\mathrm{M}$, Borgquist L, et al. Priority setting in primary health care - dilemmas and opportunities: a focus group study. BMC Fam Pract 2010;11:71.

120. Fortin M, Haggerty J, Almirall J, et al. Lifestyle factors and multimorbidity: a cross sectional study. BMC Public Health 2014;14:686.

121. Violan C, Foguet-Boreu Q, Flores-Mateo G, et al. Prevalence, determinants and patterns of multimorbidity in primary care: a systematic review of observational studies. PLoS One 2014;9:e102149.

122. Légaré F, Stacey D, Pouliot S, et al. Interprofessionalism and shared decision-making in primary care: a stepwise approach towards a new model. J Interprof Care 2011;25:18-25.

123. Dowdy D, Bishai D, Chen AH. Setting clinical priorities: a framework for incorporating individual patient preferences. Patient Educ Couns 2013;90:141-3.

124. Junius-Walker U, Wrede J, Voigt I, et al. Impact of a prioritysetting consultation on doctor-patient agreement after a geriatric assessment: cluster randomised controlled trial in German general practices. Qual Prim Care 2012;20:321-34.

125. Arvidsson E, André M, Borgquist L, et al. Setting priorities in primary health care-on whose conditions? A questionnaire study. BMC Fam Pract 2012;13:114.

126. EPOC. EPOC (Effective Practice and Organization of Care) Taxonomy. Cochrane Collaboration. http://epoc. cochrane.org/epoc-taxonomy. Published 2015 (Accessed 8th May 2017).

127. Brown S, Lhussier M, Dalkin SM, et al. Care planning: what works, for whom, and in what circumstances? a rapid realist review. Qual Health Res 2018;28:2250-66.

128. Rosbach M, Andersen JS. Patient-experienced burden of treatment in patients with multimorbidity - A systematic review of qualitative data. PLoS One 2017;12:e0179916.

129. Kinsella S. Older people and social isolation: a review of the evidence. In. Birkenhead, England: Wirral Council Business, 2014.

130. StatsCan. Seniors' income from 1976 to 2014 : Four decades, two stories. Statistics Canada. 2018 http://www.statcan.gc.ca/pub/11630-x/11-630-x2016008-eng.htm (Accessed 4 May 2018). 